\title{
AN OVERVIEW OF THE APPLICATION OF MACHINE LEARNING IN PREDICTIVE MAINTENANCE
}

\author{
Tran Ngoc Trung', Trieu Hung Truong'2, Tran Vu Tung', Ngo Huu Hai', Dao Quang Khoa' \\ 'Bien Dong Petroleum Operating Company \\ ${ }^{2} \mathrm{Ha}$ Noi University of Mining and Geology \\ Email: trungtn@biendongpoc.vn \\ https://doi.org/10.47800/PVJ.2021.10-05
}

\section{Summary}

With the rise of industrial artificial intelligence (Al), smart sensing, and the Internet of Things (IoT), companies are learning how to use their data not only for analysing the past but also for predicting the future. Maintenance is a crucial area that can drive significant cost savings and production value around the world.

Predictive maintenance (PdM) is a technique that collects, cleans, analyses, and utilises data from various manufacturing and sensing sources like machines usage, operating conditions, and equipment feedback. It applies advanced algorithms to the data, automatically compares the fed data and the information from previous cases to anticipate or predict equipment failure before it happens, thus helping optimise equipment utilisation and maintenance strategies, improve performance and productivity, and extend equipment life. Robust PdM tools enable organisations to leverage and maximise the value of their existing data to stay ahead of potential breakdowns or disruptions in services, and address them proactively instead of reacting to issues as they arise. Therefore, it has attracted more and more attention of specialists in recent years.

This paper provides a comprehensive review of the recent advancements of machine learning (ML) techniques widely applied to PdM by classifying the research according to the ML algorithms, machinery and equipment used in data acquisition. Important contributions of the researchers are highlighted, leading to some guidelines and foundation for further studies. Currently, BIENDONG POC is running some pilot PdM projects for critical equipment in Hai Thach - Moc Tinh gas processing plant.

Key words: Machine learning, predictive maintenance.

\section{Introduction}

As defined by the European Standard PN-EN 13306 [1], maintenance is "a combination of all technical, administrative, and managerial actions during the life cycle of an item intended to retain it, or restore it to a state, in which it can perform the required function". Maintenance on the field generally includes repair and replacement of equipment parts to maintain equipment within its operating conditions.

In manufacturing environment, maintenance is one of the critical success factors. Poorly maintained equipment

Date of receipt: 21/9/2021. Date of review and editing: $2 / 9$ - 16/10/2021 Date of approval: 16/10/2021. often leads to unplanned downtime and low performance. Some adverse impacts on business performance are behind schedule operation, waste increase, and poor quality products, etc. According to the International Society of Automation, the cost of machine downtime is USD 647 billion each year globally [2]. Different corporations may have different maintenance strategies depending on several factors, such as maintenance goals, equipment's nature, operational process design, and work environment. Commonly used maintenance approaches are categorised into a) corrective maintenance (CM), b) preventive maintenance $(\mathrm{PM}), \mathrm{c})$ predictive maintenance $(\mathrm{PdM})$, and d) proactive maintenance [3]. Amongst these approaches, the PdM aims to make timely maintenance decisions timely by focusing on fault detection, component diagnosis, degradation monitoring, and 
failure prediction in real-time, therefore reducing the uncertainty of maintenance activities.

With the Fourth Industrial Revolution (Industry 4.0), IT infrastructure has been continuously improved to support smart sensing, Internet of Things (IoT), big data collection, and analytics tools; companies now can get the most out of their data. Data-driven decisions can be made by analysing historical events and trends in the past [4, 5]. In the case of complex equipment with a large amount of data, processing and analysing become more and more challenging for humans to handle. There are disadvantages of human dependent scenarios, where humans monitor equipment and make decisions manually for the work needed on the equipment. First, specialists' performance is highly dependent on their expertise levels. In a study by Smith-Bindman R. et al. [6], less experienced physicians tend to make false diagnostic decisions at a rate of $50 \%$ higher than more experienced ones. This issue becomes worse with industries that lack specialists who are well trained and experienced. In addition, the consistency of diagnostic decisions among specialists cannot be guaranteed. A study by Gulshan et al. [7] compared decisions from seven US-certified ophthalmologists regarding diagnosing the severity levels of diabetic retinopathy based on photographs of the retinal fundus. It showed poor consistency among ophthalmologists, in which only $20 \%$ of the cases demonstrating a complete agreement among them. Last but not least, specialists who analyse a large number of data for a long time can make more mistakes and work less efficiently because of mental fatigue and cognitive overload [8].

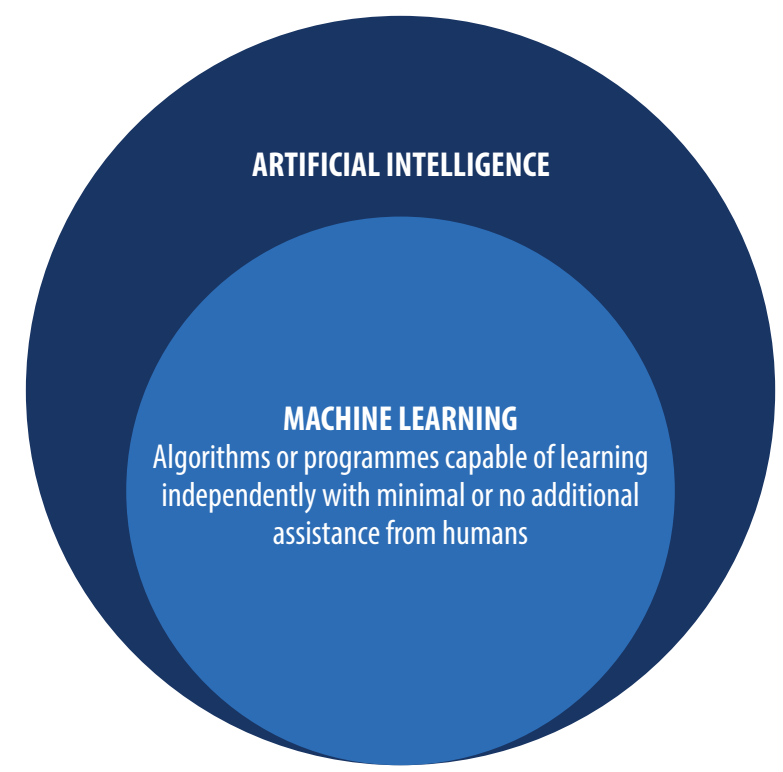

Figure 1. Machine learning is a subset of artificial intelligence.
Artificial intelligence (AI) is an element of Industry 4.0, showing its application in the manufacturing industry as a powerful tool for machine health diagnosis. A recent survey indicated that PdM is expected to be one of the first fields where Al-based technologies will be successfully implemented [9]. Indeed, it is almost impossible for a human operator to interpret data in real-time, and the conventional systems can neither spot anomalies in data nor predict a sensor reading in a specified time window, $\mathrm{Al}$ and machine learning (ML) techniques have therefore emerged as a promising tool in PdM applications for intelligent manufacturing in Industry 4.0, which involves rethinking and optimising the entire maintenance strategy as a whole.

As illustrated in Figure 1 machine learning is a subfield of $\mathrm{Al}$ and defined as an algorithm or programme capable of learning independently with minimal or without assistance from humans. ML supports solving many complex human problems, such as image processing, big data, robotics, and speech recognition. By utilising $\mathrm{ML}$ in processing, continuously monitoring, and analysing equipment's health, corporates can achieve greater operational and maintenance efficiency and effective growth management by improving equipment performance. A case study by Roosefert et al. show an impressive $84 \%$ reduction of breakdown time and $88 \%$ reduction of breakdown occurrences when applying intelligent ML-based PdM approach compared to the conventional Industry 3.0 setup [10]. This will also increase the availability and reliability of equipment and reduce operational risks, therefore improving corporates' competitive advantages. With recent advances in $\mathrm{ML}$ techniques, numerous studies have explored the potential of the ML approach and demonstrated promising results. For example, Akram et al. [11] developed the convolution neural networks (CNNs) for fault detection of photovoltaic cell defects and achieved $93.02 \%$ accuracy. In another study, Ren et al. [12] employed region-based CNN (R-CNN) to automate object detection training in realtime. Cha et al. [13] applied R-CNN to detect five types of structural surface damages on bridges and achieved a mean average precision ( $\mathrm{mAP}$ ) of $87.8 \%$. The problem of concern today regarding $\mathrm{ML}$ in $\mathrm{PdM}$ applications is to choose the most suitable, simple, and effective algorithm for each specific problem. ML algorithms often require large data acquisition about failure situations and different operating states of the system or device to train the model. 


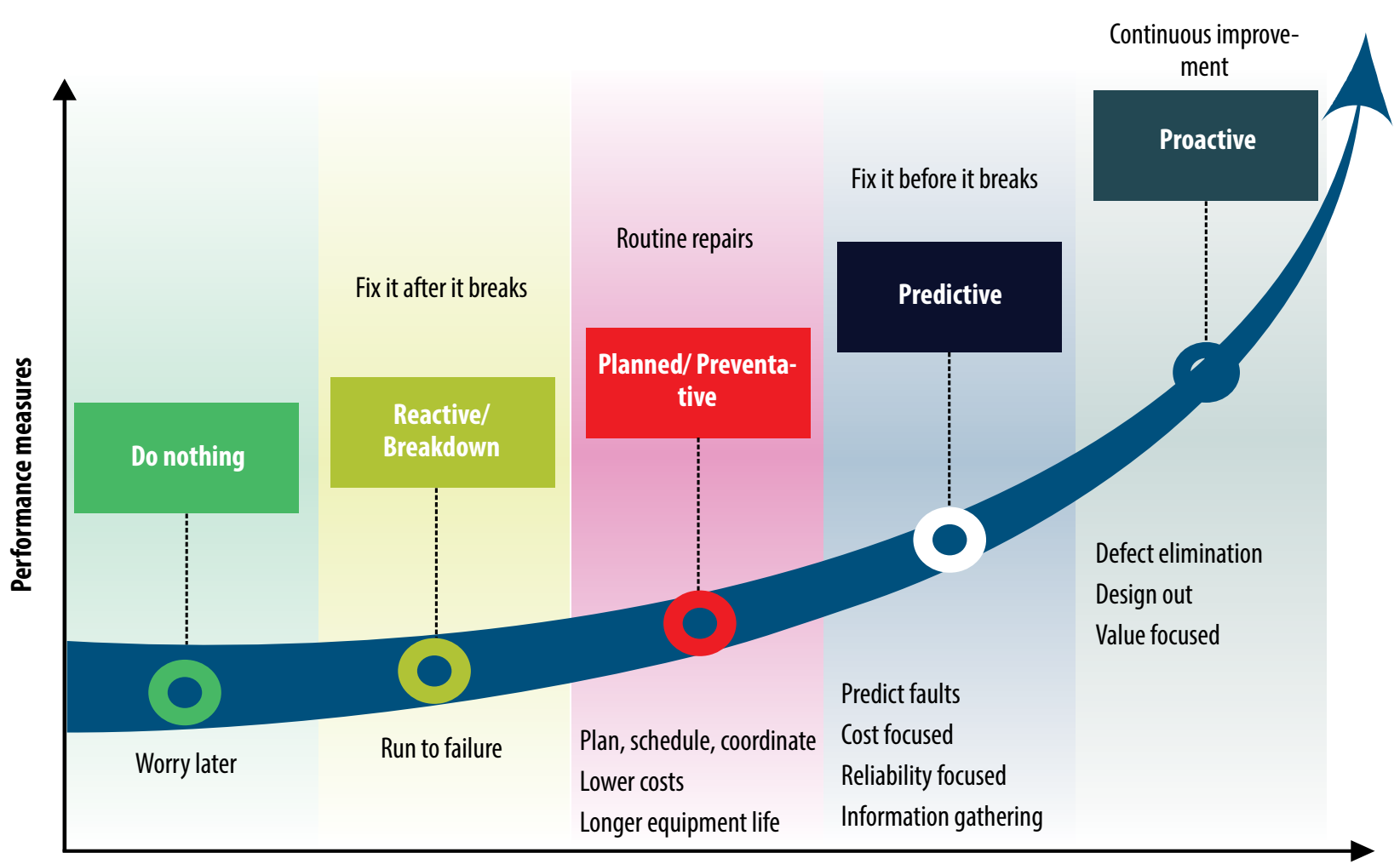

Figure 2. Evolution of maintenance approaches [3].

\section{Evolution of maintenance approaches}

The evolution of maintenance approaches is presented in Figure 2.

Corrective maintenance (CM), also known as "breakdown maintenance" or "run to failure", is the first approach to maintenance. No routine maintenance activity is scheduled in this approach, and maintenance actions only occur after equipment breakdown. It is, therefore, impossible to optimise equipment performance with respect to economic or reliability criteria [3], and subsequently, equipment availability and reliability performance are substandard. Despite being inferior to other approaches, the CM approach is still in use due to the low cost of implementation, and suitable for equipment that has a low budget to repair or replace. Companies often apply $\mathrm{CM}$ to equipment that has insignificant or no impact on the whole production line.

Preventive maintenance (PM) is the next stage of maintenance approach evolution. PM is a time-based approach where inspection and replacement/repair works are scheduled and conducted regularly before any failure can happen. It aims to avoid system failures during operation, especially when such an event is costly and/or dangerous. PM is the dominant maintenance policy used in industry. For systems such as transportation, production, or critical infrastructure, the time-based inspection and maintenance policies can improve performance, increase reliability and capability of assets concerned, and reduce the cost of assets running [14]. PM, however, is not always cost-effective. Because maintenance work is carried out on a time basis regardless of equipment's conditions, it can lead to a high workload and cost if the equipment is still working correctly. Another disadvantage of PM is the infant mortality of equipment induced by human error during preventive maintenance work.

The next level of maintenance approach evolution is condition-based maintenance (CBM), and its improved version which is the so-called predictive maintenance (PdM). The PdM approach uses advanced analytics techniques to predict faults or failures in a deteriorating system to optimise maintenance efforts by monitoring equipment operating conditions and performances to detect any signs of wear or deterioration leading to a component's failure $[15,16]$. More than just monitoring the basic condition of equipment, the main objective of PdM which is to predict the remaining useful life of machines using historical data is one of the promising areas of machine prognosis which could lead to significant maintenance optimisation and cost savings. 
PdM approaches have been extensively applied in industries for handling the health status of equipment by detecting early signs of failure in advance, enabling maintenance measures to be taken ahead of time, which allows the saving of more costs because repair after failure is always more expensive than maintenance in advance [17]. According to data from McKinsey [18], PdM tools can reduce manufacturing machine downtime by $30 \%-50 \%$ and increase machine life by $20 \%-40 \%$. Besides, PdM initiatives enable organisations around the world to save USD 17B in 2018 globally [19].

In PdM, the optimal time point for maintenance actions is predicted by analysing the system's health state and historical maintenance data to make a timely repair to avoid costly repairs due to system breakdown and premature maintenance activities which may induce infant mortality. The PdM approach is used in industrial sectors where reliability is paramount, like nuclear power plants, transportation systems, or emergency systems [3]. The most used monitoring and diagnostic techniques include vibration monitoring, thermography, tribology, and visual inspection.

On the other hand, proactive maintenance emphasises keeping assets and equipment in top conditions by identifying potential problems and addressing them as early as possible. That is one of the main reasons why proactive maintenance is considered different from other approaches that help reduce maintenance costs and risk of property damage. Some examples of proactive maintenance activities are: (i) applying anti-corrosion coating to metal surfaces; (ii) performing an inspection for cracks, leaks, and rust; (iii) lubricating the machine to reduce wear and diffusion corrosion and (iv) applying sealant for junction boxes to prevent water ingress. In proactive maintenance approach, possible causes of equipment failure are analysed, and works are carried out to eliminate those causes in very early stages (e.g., designing, installation, and commissioning stages), which definitely distinguish proactive maintenance from other approaches.

\section{ML approach in PdM}

In PdM approach, equipment health is continuously monitored and analysed in real-time. Lei et al. [20] divide the process of diagnosing heath of machinery into four steps: (i) data acquisition; (ii) health indicators construction; (iii) health stage division; and (iv) remaining useful life prediction.
In data acquisition phase, the measured data, such as vibration, current, temperature, or pressure signals, are collected from sensors to monitor the health condition of machinery. In the next step, health indicators (HI) are constructed to represent the health condition of machinery using statistics, signal processing, or artificial intelligence techniques. Then, the $\mathrm{HI}$ will be analysed and classified into two or more health stages (HS) depending on $\mathrm{HI}$ degradation trends. In the final step, the remaining useful life is predicted to determine how much time is left until machinery reaches the end of its lifetime, based on which optimal maintenance activities can be scheduled.

\subsection{Data acquisition}

Data acquisition is a prerequisite from which raw data can be processed and analysed later to solve a PdM problem. Data acquisition involves designing and sizing the appropriate system architecture to be installed on the equipment to capture and store different sensing data. A data acquisition system consists of sensors, data transmission, and data storage devices. Depending on equipment types, different combinations of sensors are used so that the captured data can fully reflect the degradation process of machinery. Some commonly used sensors are accelerometers, acoustic emission sensors, infrared thermometers, current, temperature, and pressure sensors, etc. The captured data are transmitted into a PC or portable devices through a data transmission device and stored in a memory location or historian system for further analysis. With a rapid development of sensor and communication technologies, more advanced data acquisition devices have been designed and applied to modern industries.

One of the essential data acquisition techniques often discussed in ML algorithms is data pre-processing techniques. In this step, commonly used techniques for data pre-processing include data normalisation and noise removal, feature extraction, and feature selection. Feature extraction is the process of mapping the original dataset to new data space, including new attributes that contain more explicit information for performing PdM. There are two basic feature extraction methods commonly applied in PdM: data-driven and statistical techniques.

With the data-driven technique, these feature extraction processes are performed automatically based on specific data sets (data-driven) using a machine learning model. The weakness of this approach is that a lot of data will be needed to train the model, and the 
user may not be able to be interpreted or described the extracted attributes. Several proven examples of machine learning tools used for feature extraction include CNN [21, 22], autoencoder [22 - 24], or principal component analysis [25 - 27].

Feature extraction based on statistical theory is a classic and commonly used method in data studies. Statistical methods will usually be used first in surveying and understanding system performance trends from sensor data. Statistical methods can be performed in three types [27]: time domain, frequency domain, and time-frequency domain. Statistical methods in the time domain include monotony, trend, consistency, similarity, stability, or correlation. Many techniques can be used to extract these features, including peak value, the difference between the highest and the lowest values, mean absolute value, root mean square (RMS), crest factor, standard deviation, kurtosis, or distributed shape factor. The feature extraction of the time domain technique have many advantages - it is fast, simple, and can be used for many different types of device failures. The disadvantage of time-domain feature extraction is that it is susceptible to noise and often requires data pre-processing before extraction. The advantage of feature extraction in the frequency domain technique is its efficiency in representing anomaly information and equipment errors, which are not visible in the time domain. The disadvantage of the frequency domain technique is the need for specialised knowledge of device failures in the frequency domain, and this method cannot be universally applicable to all types of devices. The time-frequency domain technique is a powerful tool that allows analysing and representing essential features of the data spectrum over time. Time-frequency domain feature extraction method is generally not affected by noise. However, some of its disadvantages are high computational cost, and the difficulty in determining the parameters of the data transformation process from the original time domain to the time-frequency domain. Statistical approaches have been widely applied in predictive intelligence problems and achieved many positive results [28, 29].

After collecting these values, we can do one more step called feature fusion or feature aggregation. This step combines the features extracted from the above statistical method to create more complex features with better data representation. For example, we can use other mathematical formulas [28] or genetic algorithms [29] to combine features.

\subsection{Health indicator construction}

After extracting the features, the next step is to determine the health indicator. Indeed, a health indicator represents the health state of machinery in real-time, taking into account different kinds of condition monitoring signals, such as vibration, current, and acoustic emission signals. $\mathrm{HI}$ construction plays a significant role in PdM. A suitable $\mathrm{HI}$ can help simplify the prognostic modelling and produce a more accurate prediction. In addition, from the $\mathrm{HI}$ information, specific methods can be devised for estimating the remaining useful life of the device. HI can be derived from monitoring signals using statistical methods or signal processing methods, such as RMS of vibration signals. In fact, machines are often monitored by multiple sensors. While analysing a high-dimensional dataset, different Al techniques can be employed to fuse those multi-sensor signals into an indicator representing the machinery's degradation trend.

In a study by Lei et al. in 2018 , there are two main techniques to develop the $\mathrm{HI}$ [30], including (i) physical $\mathrm{HI}(\mathrm{PHI})$ relating to physical faults of the machine and usually extracted from the sensor using statistical or data processing techniques; (ii) virtual $\mathrm{HI}(\mathrm{VHI})$ built by combining many physical indicators or the different sensors. VHI usually carries no physical implication about the device but only information about the failure tendency of the device in a virtual manner.

Principal component analysis (PCA) is one of the most popular techniques for $\mathrm{HI}$ construction. PCA is a linear dimension reduction technique that uses an orthogonal transformation to convert a set of observations of possibly correlated or dependent variables into a set of linearly uncorrelated variables called principal components. Some of the significant studies are the construction of an $\mathrm{HI}$ using PCA to reduce the dimensions of the feature sets and subsequently calculate the deviations between unknown states and the healthy state [25], using PCA combined with isometric feature mapping to construct an $\mathrm{HI}$ for cutting tools [26], and fusing multiple features to calculate the $\mathrm{T}^{2}$ statistics as an $\mathrm{HI}$ of bearings [27].

Self-organising map (SOM) is another widely used technique for HI construction. The SOM is a nonsupervised learning neural network that produces a low-dimensional representation of a higher-dimensional dataset by organising itself according to the nature of the input data. The SOM technique was introduced into $\mathrm{HI}$ construction by Qiu et al. [31] for the condition 
monitoring of roller bearing. Some authors also used the SOM technique for $\mathrm{HI}$ construction. Hong et al. [32] used wavelet packet decomposition (WPD) and empirical mode decomposition (EMD) to extract entropy sequences from original vibration signals and use them as input vectors of the SOM network. The confidence value derived from the SOM is then used as an HI of bearing. Lei et al. [33] trained the SOM by the feature vector in the operational stage. Then the feature vector under an unidentified condition is compared with the weight vector of its best matching unit (BMU) in the SOM. Liao et al. [34] used the Restricted Boltzmann Machine algorithm to extract features from the dataset and compare them with the weight vectors of all the units in the baseline map. The distance to the baseline map is calculated as minimum quantisation error (MQE), used as the machine's health value. And Huang et al. [35] trained the SOM network using 6 vibration features and used the MQE indicator derived from SOM as an $\mathrm{HI}$.

Mahalanobis distance (MD) is used in some publications as an $\mathrm{HI}$ construction technique. MD generalises multivariate dataset by finding how many standard deviations away a point is from the mean of the multivariate distribution. Wang et al. [36] utilised Mahalanobis distance to construct $\mathrm{HI}$ from 14 different statistics into a new feature to reflect the degradation of the bearing. Jin et al. [37] calculated the energies of wavelet coefficients and fused them by calculating their Mahalanobis distance with reference to the healthy one. Kumar et al. [38] also constructed an $\mathrm{HI}$ based on Mahalanobis distance to monitor bearings.

Researchers also explored different combinations of Al techniques for $\mathrm{HI}$ construction. Ocak et al. [39] used the wavelet packet decomposition (WPD), a time-frequency domain technique, in connection with the hidden Markov modelling (HMM) technique to develop a method for real-time tracking of bearing health and prognostic. HHM has also been successfully applied to many other fields, such as tool wear condition monitoring [40] and bearing diagnosis [41]. Shen et al. [42] constructed an $\mathrm{HI}$ that reflects the running state of the rolling bearing in realtime and effectively guarantees the operation reliability of bearings using the fuzzy support vector data description (FSVDD) technique. Liu et al. [43] proposed an $\mathrm{HI}$ for bearings through phase space reconstitution combined with approximate diagonalisation of eigen-matrices. Guo et al. [44] constructed an $\mathrm{HI}$ that contains rich degradation signatures of bearings from six related-similarity features and eight time-frequency features using the recurrent neural network.

Besides using mathematical functions to define $\mathrm{HI}$, another way is to compare the current state of the device to be monitored to that of a similar one. The selected devices are also working in the same environment and performing the same task. When the operating envelope of the two devices is the same, their $\mathrm{HI}$ will be the same.

\subsection{Health stage division}

Staged machine operation is the next step after determining $\mathrm{HI}$. However, it is not always easy to separate the working states of the machine. In particular, if the device continuously works with a linear $\mathrm{HI}$ from start to finish, it is impossible to subdivide the device's different working stages. In that case, the working state of the device changes uniformly at a constant rate [30]. However, it does not mean that this is an unimportant step in the PdM application. With many other types of mechanical devices (such as journal bearings), the operating state of the equipment can be divided into two or more stages. With these devices, in the healthy stage, the $\mathrm{HI}$ readings are nearly constant, so there is no information about the failure trend of the device. Forecasting the RUL at this stage would be neither accurate nor necessary. RUL forecasting should only begin to be performed after the machine transitions from normal operation to deterioration due to failure or a so-called unhealthy state. Then, the division of working states makes sense and is used to determine the device's initial degradations and provide a reasonable transition time for the RUL estimation in PdM.

HIs of machinery generally present varying degradation trends with the development of fault severity. Subsequently, the degradation process of machinery can be divided into different health stages (HS) according to the varying trends of HI. HS division is vital in machinery health diagnostics because it identifies the start time of the unhealthy stage, also known as the first predicting time (FPT), and triggers the remaining useful life (RUL) prediction [20].

In one-stage HS, the health of machinery shows gradual degradation, which can be described using a single degradation model. In this case, the HS division process is not required. In two-stage $\mathrm{HS}$, the degradation trend shows two distinct stages: the healthy stage, where no fault occurs, and the unhealthy stage, where there is an accelerated degradation trend over time. In multiple-stage HS, the 
unhealthy equipment stage is further divided into different stages according to the characteristics of degradation trends. HS division can classify the degradation process into one stage, two stages, multiple stages (Figure 3).

The most straightforward approach in HS division is to identify whether the $\mathrm{HI}$ exceeds a constant alarm threshold. However, using a constant alarm threshold may yield false alarms due to random noise interference [20]. Some publications used a clustering algorithm to derive an adaptive threshold to avoid this problem. A commonly used algorithm is K-nearest neighbor (KNN), a data classification approach that estimates how likely a data point is to be a member of one group or the other depending on what group the data points nearest to it are in a study by Ramasso et al. [44]. KNN is used to assess the system's discrete state (functioning mode) while using a multidimensional degradation signal. Other Al classification algorithms are also studied in different publications. Kamran et al. [46] developed a model using a fuzzy c-mean clustering algorithm that can adaptively estimate the health state of the system rather than with a fixed number of states. Liu et al. [47] trained a model by applying PCA to reduce the dataset dimension and then using fuzzy c-means to automatically extract knowledge about health state labels of all the time points. Scanlon et al. [48] used a K-means clustering algorithm to determine the boundaries between different states.

The multi-stage degradation processes can also be described using discrete state transition models such as the hidden Markov model (HMM). The hidden Markov model is a probabilistic model attempting to explain or derive the probabilistic characteristic of any random process. It says that an observed event will not correspond to its step-

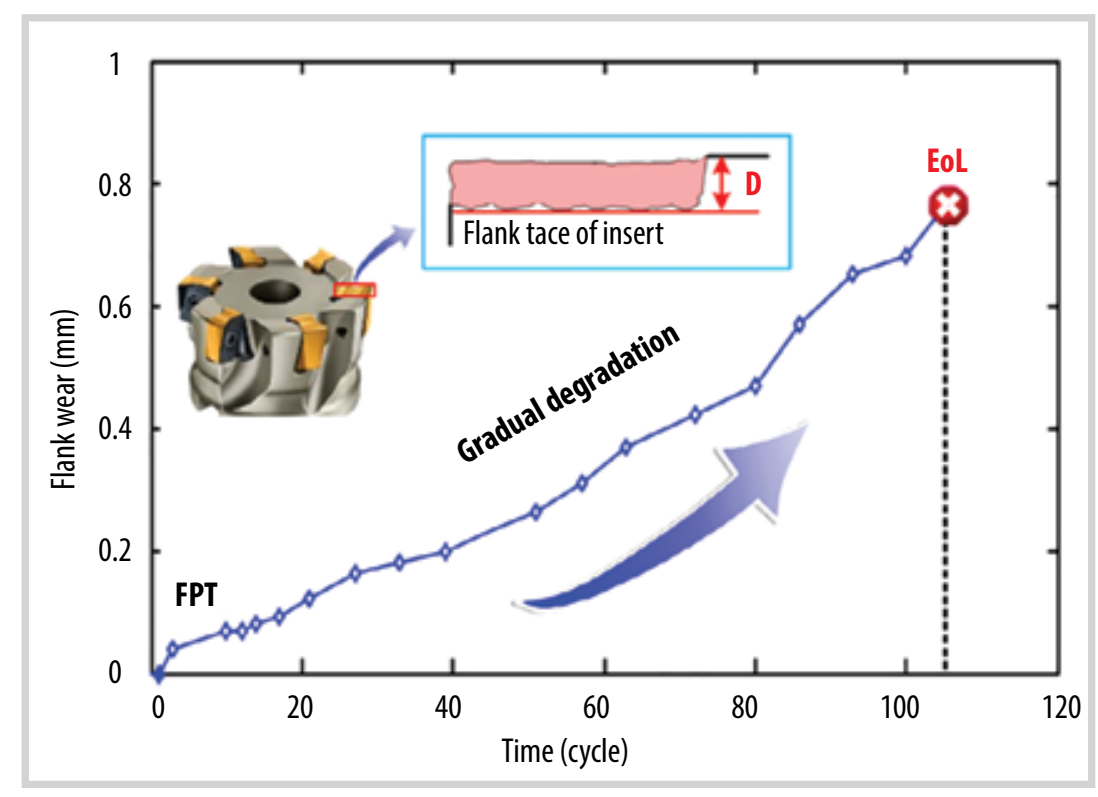

(a)

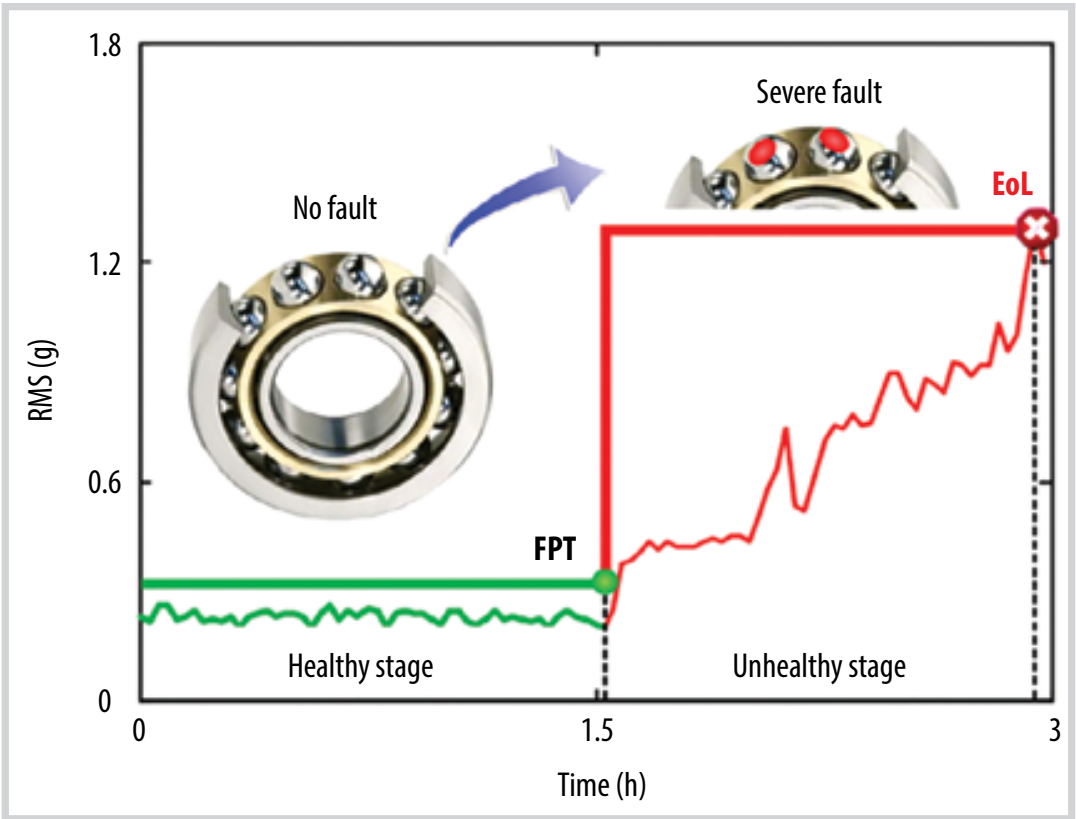

(b)

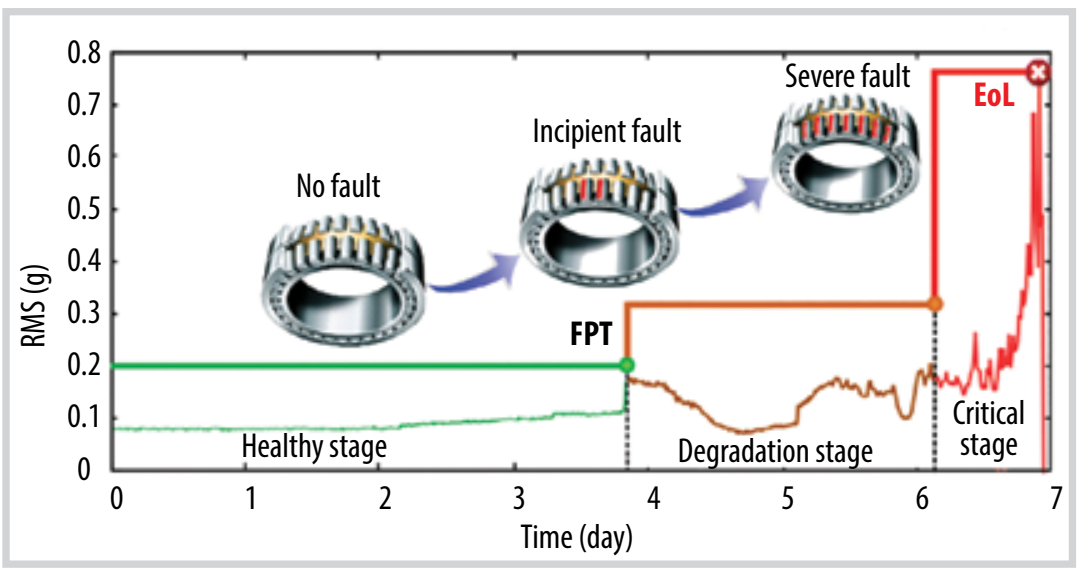

(c)

Figure 3. Degradation processes with (a) one stage, (b) two stages, and (c) multiple stages [20]. 
by-step status but a set of probability distributions. HMM is also applied in the studies of Giantomassi et al. [49], Ramasso et al. [50], and Sloukia et al. [51]. Some AI classifiers are applied to multi-stage HS division as well, such as artificial neural networks [52 - 54] and support vector machine $[55,56]$.

\subsection{Remaining useful life prediction}

Prediction of the remaining useful life (RUL) of machinery is the most critical problem in the field of PdM. The RUL of machinery is defined as "the length from the current time to the end of the useful life" [57]. The primary task of RUL prediction is to forecast the time left before the machinery loses its operation ability based on the condition monitoring information. It is the last technical process as well as the last goal of machinery prognostics. Approaches for RUL prediction can be categorised into physical model-based approaches, statistical modelbased approaches, and Al approaches.

Physical model-based is a classical approach that describes degradation processes of machinery through building mathematical models based on the failure mechanisms or the first principal damage [58]. The parameters of the physical models reflect the material properties and stress levels, which are generally obtained by experiments or finite element analysis. To provide an accurate estimation of the RUL physical model-based approach, it is required that the model is developed with a complete understanding of the failure mechanisms and effective estimation of model parameters. However, it is difficult for some complex mechanical systems to understand the physics the damage, which restricts the application of these approaches [20].

Statistical model-based approaches, also known as empirical model-based approaches, estimate the RUL of machinery by establishing statistical models based on empirical knowledge [57]. The statistical model-based approach does not rely on physics to construct RUL prediction models. Rather, the RUL is predicted by fitting available observations into random coefficient models or stochastic process models under a probabilistic method. In the RUL problem, this method uses probabilistic statistical models to represent the relationship between variables in the state of the device. Random variances are generally introduced into model parameters to describe the uncertainties caused by different kinds of variability sources, such as temporal variability, unit-to-unit variability, and measurement variability [59]. Therefore, the statistical model-based approaches effectively describe the uncertainty of degradation process and its influence on RUL prediction.

Al approaches attempt to learn the machinery degradation patterns using Al techniques from available observations instead of building physical models or statistical models. They can deal with prognostic issues of complex mechanical systems whose degradation processes are challenging to be interrelated by physical models or statistical models. Therefore, they are attracting more and more attention in the field of machinery prognostics. The results of $\mathrm{Al}$ approaches are hard to explain because of the lack of transparency; thus, these techniques are consistently named "black boxes" [20]. The commonly used $\mathrm{Al}$ techniques in machinery prognostics include artificial neural networks, neuro-fuzzy systems, support vector machine, K-nearest neighbour, and Gaussian process regression (GPR), etc.

The artificial neural network is an ML reasoning technique inspired by the working process of human brains. It is a collection of nodes, also known as artificial neurons, in a complex structure of the input, hidden, and output layers. In ANNs, different layers may perform different transformations on their inputs. Input layer nodes pass information to hidden layer nodes by activation functions. Subsequently, the hidden layers apply weighting functions until some threshold of the hidden layer is reached. Finally, the result is passed to the output layer. Because ANNs can learn complex non-linear relationships by training the multi-layer networks, they have a good performance in dealing with complex systems and become the most used Al techniques in machinery RUL prediction. Two widely used ANNs are feed-forward neural network and recurrent neural network, which are classified based on the architecture of layers in the network. The difference is illustrated in Figure 4.

The feed-forward neural network (FFNN) is a class of ANNs, where inputs are processed forward. Sbarufatti et al. [60] combined FFNNs with sequential Monte-Carlo sampling to predict the RUL of fatigue cracks. Pan et al. [61] and Xiao et al. [62] used an FFNN to conduct multi-step ahead prediction for the bearing health states. Wang et al. [63] used a three-layer FFNN to predict the future HIs and input the predicted HIs into a $\mathrm{PH}$ model to estimate the hazard rate and survival probability. The results showed that RUL can be predicted with high accuracy in the 
incipient degradation phase and trace the degradation well with a limited amount of data.

On the other hand, the recurrent neural network (RNN) is another class of ANNs that have many applications in solving time-series or sequential data problems. Unlike FFNN, which assumes inputs and outputs are independent, RNN has a recurrent connection in the hidden state that makes the output of RNN depend on the prior or previous elements within the sequence. RNN method is widely used in RUL prediction because of its ability to deal with detailed time-series data. As illustrated in many studies, this method showed good performance, such as the recurrent radial basis function network to predict the RUL of machinery by Zemouri [64]. Malhi et al. developed a competitive learning-based approach to revise the training technique of RNNs that helps improve the long-term prediction accuracy [65]. In addition, Peng et al. improved the RNN model by replacing a hidden layer using a large sparse reservoir to develop a new RUL prediction approach [66]. Liu et al. proposed an enhanced RNN for RUL prediction by improving RNNs' memory property [67].

The ANN networks still have limitations in terms of their low transparency and the requirement of a large dataset for high-quality training process, which are difficult to capture in industrial applications. In addition, their structures and parameters are generally initialised randomly or specified manually, which reduces their generalisation ability among different cases [20].

The neural fuzzy (NF) approach is based on the

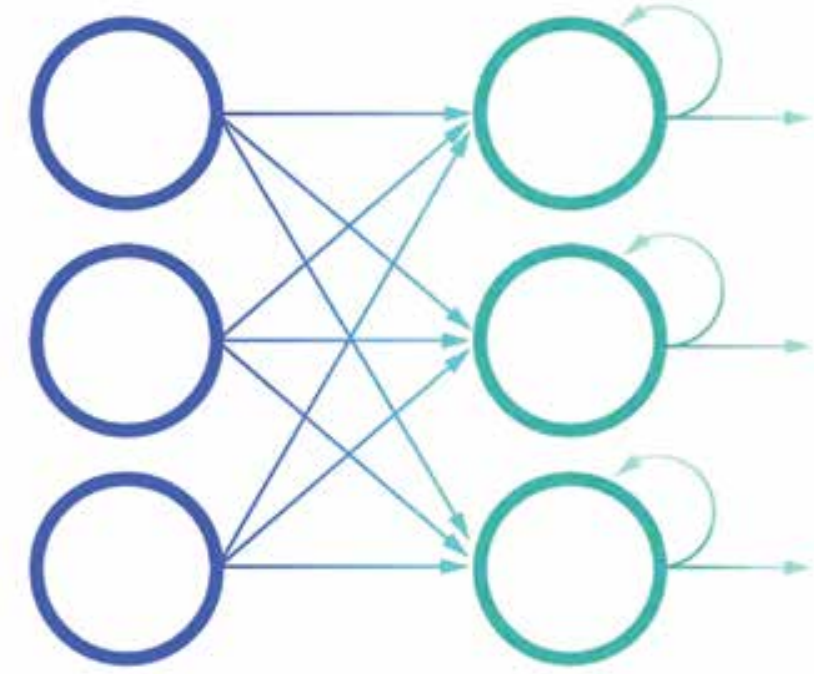

(a)

Figure 4. Comparison of RNN (a) and FFNN (b) [68]. fuzzy system trained by algorithms derived from neural networks. The basic idea behind this NF system is that it combines the human-like reasoning style of fuzzy systems with neural networks' learning and connectionist structure [69]. The NF-based time-series forecasting approach was proposed by Jang et al. [70]. Wang et al. [71] adopted the NF system to develop an online prognostic approach for different kinds of gear faults. Wang [72] further improve this approach from several aspects to enhance its forecasting performance. NF systems take advantage of both the expert knowledge and the intelligent ANNs, thus being competitive candidates for machinery RUL prediction [73 - 77]. However, they still need lots of highquality training data.

Support vector machine (SVM) is a kind of $\mathrm{ML}$ technique based on the statistical learning theory proposed by Vapnik [78]. Different kinds of SVM have been applied to the RUL prediction of machinery, such as the least square-SVM [79], one-class SVM [80], and multiclass SVM [81]. Widodo et al. [82] trained an SVM model using both the censored and the complete data and predicted the survival probability of machinery. Tran et al. [83] integrated an SVM-based RUL prediction module into an intelligent condition-based maintenance platform. In addition, support vector regression (SVR) is the standard application form of SVM in prognostics [83]. Benkedjouh et al. [84] used the SVR to map the HIs into non-linear regressions and then fitted the obtained regressions into power models for RUL prediction of machinery. Liu et al. [85] developed a modified probabilistic SVR to predict the degradation processes of nuclear power plant

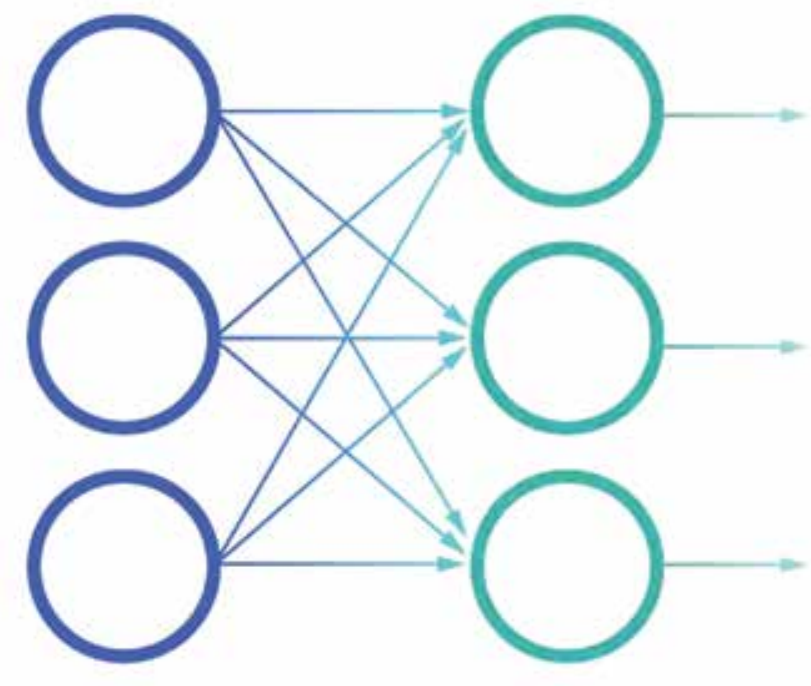

(b) 
components. Fumeo et al. [86] developed an online SVR model for the RUL prediction of bearings by optimising the trade-off between accuracy and the computing efficiency.

Compared to ANNs, SVM are superior in dealing with the issues of small sample sizes. Thus, they may be more suitable for the issues of RUL prediction where only limited measurements are available. However, the performance of SVM is highly dependent on the selected kernel functions and parameter optimisation process.

Gaussian process regression (GPR) is an $M L$ technique that implements Gaussian processes for regression purposes [87]. Gaussian processes are cumulative damage processes of random variables with joint multivariate Gaussian distributions. Many regression models have been published recently, such as introducing theoretical details and the flexibility of the GPR in non-linear regression [88], predicting RUL by GPR [89], applying GPR with three different covariance functions for RUL prediction of bearings [32] and predicting the degradation trends of rolling element bearings using an integrated GPR model [90]. In contrast to the above Al techniques, GPR is highly adaptable and suitable for RUL prediction problem of high-dimension and small-size datasets [91]. The major drawback of GPR is that it generally requires heavy computational work.

\subsection{Challenges for PdM using ML in Vietnam}

In essence, each device has many different failure modes and must be tested in laboratory to produce the most complete and standard datasets. During normal operating conditions, these figures are difficult to obtain. There can only be specific failure modes. In addition, it is necessary to aggregate data on equivalent machines. It is challenging to compare and synthesise data in many cases due to the unavailability of machines in most companies. Where possible, oil and gas companies, especially in Vietnam, need to collaborate and share data about machinery to support the development and research of PdM.

Infrastructure for data acquisition is not enough due to high installation and maintenance costs. Instrumentation is often poorly equipped or does not have a proper acquisition system to collect data for a long time. Moreover, special data acquisition equipment such as vibration analyser, ultrasonic sound measurement, and oil sampling analyser is typically expensive, dependent on advanced technology or requiring intensive training to use. Recently, PdM applications often used Al and ML. Due to the lack of specialised research facilities for PdM in Vietnam, there will be many difficulties and challenges for the technology to be applied in the country. However, the benefits it brings if successfully implemented are very desirable.

\section{Conclusion}

PdM practices become more and more popular in many industrial sectors because of their effectiveness in reducing unnecessary maintenance operations and improving machinery reliability. With the recent advancement of Industry 4.0, ML has found wide applications in PdM practice to assist humans in processing, continuously monitoring and analysing equipment's health. This paper has discussed the practice of PdM and reviewed the applications of some state-ofthe-art Al techniques that have been studied in this field. Different Al techniques are structurally discussed in the four technical steps of the machinery health prognostics process, including data acquisition, $\mathrm{HI}$ construction, HS division, and RUL prediction.

BIENDONG POC is aiming to deploy PdM solutions for monitoring, maintaining, and tracking equipment's reliability and performance in a plant by $\mathrm{ML}$ modelling. The monitoring and quick identification of failures help make decisions and plans for maintenance and enable support for continuous performance, health surveillance, and focus on performance degradation as a leading indicator of potential equipment problems. Therefore, it is necessary to review research achievements and trends in $\mathrm{PdM}$ to assess the current status and orientate the focus of research at BIENDONG POC.

\section{Acknowledgement}

This research work is described herein as part of the government research project number 077.2021.CNKK. QG/HĐKHCN, order 196/QD-BCT of Vietnam Ministry of Industry and Trade, Viet Nam.

\section{References}

[1] BS EN 1336:2017, "Maintenance - Maintenance terminology", 2017.

[2] Raghu Raj, "How preventive maintenance can backfire and harm your assets", 2020. [Online]. Available: 
https://www.ey.com/en_gl/consulting/how-preventivemaintenance-can-backfire-and-harm-your-assets.

[3] Tomasz Nowakowski, Agnieszka Tubis, and Sylwia Werbińska-Wojciechowska, "Evolution of technical systems maintenance approaches-review and a case study", International Conference on Intelligent Systems in Production Engineering and Maintenance. Springer, 2018, pp. 161 - 174.

[4] Tran Vu Tung, Tran Ngoc Trung, Ngo Huu Hai, and Nguyen Thanh Tinh, "Digital transformation in oil and gas companies - A case study of Bien Dong POC", Petrovietnam Journal, Vol. 10, pp. 67 - 78, 2020. DOI: 10.47800/ PVJ.2020.10-07.

[5] Trần Ngọc Trung, Trần Vũ Tùng, Hoàng Kỳ Sơn, Ngô Hữu Hải và Đào Quang Khoa, "Thực tiễn triển khai nền tảng số hóa tập trung tại mỏ Hải Thạch - Mộc Tinh", Tạp chí Dâu khí, Số 12, trang 47 - 56, 2020. DOI: 10.47800/ PVJ.2020.12-06.

[6] Rebecca Smith-Bindman, Philip Chu, Diana Lynn Miglioretti, Chris Quale, Robert Daniel Rosenberg, Gary Cutter, Berta Geller, Peter Bacchetti, Edward A. Sickles, and Karla Kerlikowske, "Physician predictors of mammographic accuracy", Journal of the National Cancer Institute, Vol. 97, No. 5, pp. 358 - 367, 2005. DOI: 10.1093/ jnci/dji060.

[7] Varun Gulshan, Lily Peng, Marc Coram, Martin C. Stumpe, Derek Wu, Arunachalam Narayanaswamy, Subhashini Venugopalan, Kasumi Widner, Tom Madams, Jorge Cuadros, Ramasamy Kim, Rajiv Raman, Philip C. Nelson, Jessica L. Mega, and Dale R.Webster, "Development and validation of a deep learning algorithm for detection of diabetic retinopathy in retinal fundus photographs", JAMA, Vol. 316, No. 22, pp. 2402 - 2410, 2016. DOI: 10.1001/ jama.2016.17216.

[8] Bruce I. Reiner and Elizabeth Krupinski, "The insidious problem of fatigue in medical imaging practice", Journal of Digital Imaging, Vol. 25, No. 1, pp. 3 - 6, 2012. DOI: 10.1007/s10278-011-9436-4.

[9] Won Shin, Jeongyun Han, and Wonjong Rhee, "Al-assistance for predictive maintenance of renewable energy systems", Energy, Vol. 221, 2021. DOI: 10.1016/j. energy.2021.119775.

[10] Roosefert Mohan T., Preetha Roselyn J., Annie Uthra R., Devaraj D., and Umachandran K., "Intelligent machine learning based total productive maintenance approach for achieving zero downtime in industrial machinery", Computer \& Industrial Engineering, Vol. 157, 2021.

[11] M.Waqar Akramad, Guiqiang Li, Yi Jin, Xiao Chen, Changan Zhu, Xudong Zhao, Abdul Khaliq, M.Faheem, and Ashfaq Ahmad, "CNN based automatic detection of photovoltaic cell defects in electroluminescence images", Energy, Vol. 189, 2019. DOI: 10.1016/j.energy.2019.116319.

[12] Shaoqing Ren, Kaiming He, Ross Girshick, and Jian Sun, "Faster R-CNN:Towards real-time object detection with region proposal networks", IEEE Transactions on Pattern Analysis and Machine Intelligence, Vol. 39, pp. 11371149, 2017. DOI: 10.1109/TPAMI.2016.2577031.

[13] Young-Jin Cha, Wooram Choi, Gahyun Suh, Sadegh Mahmoudkhani, and Oral Büyüköztürk, "Autonomous structural visual inspection using regionbased deep learning for detecting multiple damage types", Computer-Aided Civil and Infrastructure Engineering, Vol. 33, No. 9, pp. 731 - 747, 2018. DOI: doi/10.1111/ mice.12334.

[14] Wenbin Wang, Matthew J. Carr, Tommy Wai Shing Chow, and Michael G. Pecht, "A two-level inspection model with technological insertions", IEEE Transactions on Reliability, Vol. 61, pp. 479 - 490, 2012. DOI: 10.1109/ TR.2012.2183911.

[15] Sule Selcuk, "Predictive maintenance, its implementation and latest trends", Journal of Engineering Manufacture, Vol. 231, No. 9, pp. 1670 - 1679, 2017. DOI: $10.1177 / 0954405415601640$.

[16] R. Keith Mobley, Lindley R. Higgins, and Darrin $J$. Wikoff, Maintenance engineering handbook, $7^{\text {th }}$ edition. McGraw-Hill Education, 2008.

[17] PwC, "Predictive maintenance 4.0 - Beyond the hype: PdM 4.0 delivers results", 2018. [Online]. Available: https://www.pwc.de/de/industrielle-produktion/pwcpredictive-maintenance-4-0.pdf.

[18] Valerio Dilda, Lapo Mori, Olivier Noterdaeme, and Christoph Schmitz, "Manufacturing: Analytics unleashes productivity and profitability", 2017. [Online]. Available: https://www.mckinsey.com/businessfunctions/operations/our-insights/manufacturinganalytics-unleashes-productivity-and-profitability.

[19] Knud Lasse Lueth, "Predictive maintenance initiatives saved organizations $\$ 17 B$ in 2018 , as the number of vendors surges", 2019. [Online]. Available: https:// 
iot-analytics.com/numbers-of-predictive-maintenancevendors-surges/.

[20] Yaguo Lei, Naipeng Li, Liang Guo, Ningbo Li, Tao Yan, and Jing Lin, "Machinery health prognostics: A systematic review from data acquisition to RUL prediction", Mechanical Systems and Signal Processing, Vol. 104, pp. 799 - 834, 2018. DOI: 10.1016/j.ymssp.2017.11.016.

[21] Rui Zhao, Ruqiang Yan, Jinjiang Wang, and Kezhi Mao, "Learning to monitor machine health with convolutional bi-directional LSTM networks", Sensors, Vol. 17, No. 2, pp. 273, 2017. DOI: 10.3390/s17020273.

[22] Ling Xiang, Penghe Wang, Xin Yang, Aijun Hu, and Hao Su, "Fault detection of wind turbine based on SCADA data analysis using CNN and LSTM with attention mechanism", Measurement, Vol. 175, No. 8, 2021. DOI: 10.1016/j.measurement.2021.109094.

[23] Pankaj Malhotra, Lovekesh Vig, Gautam Shroff, and Puneet Agarwal, "Long short term memory networks for anomaly detection in time series", 23 $3^{\text {rd }}$ European Symposium on Artificial Neural Networks, Computational Intelligence and Machine Learning (ESANN), Bruges, Belgium, 2015.

[24] Pankaj Malhotra, Anusha Ramakrishnan, Gaurangi Anand, Lovekesh Vig, Puneet Agarwal, and Gautam Shroff , "LSTM-based encoder-decoder for multisensor anomaly detection", 2016. [Online]. Available: https://arxiv.org/pdf/1607.00148.pdf.

[25] Achmad Widodo and Bo-Suk Yang, "Application of relevance vector machine and survival probability to machine degradation assessment", Expert Systems with Applications, Vol. 38, No. 3, pp. 2592 - 2599, 2011. DOI: 10.1016/j.eswa.2010.08.049.

[26] Tarak Benkedjouh, Kamal Medjaher, Noureddine Zerhouni, and Said Rechak, "Health assessment and life prediction of cutting tools based on support vector regression", Journal of Intelligent Manufacturing, Vol. 26, No. 2, pp. 213 - 223, 2015. DOI: 10.1007/s10845-013-07746.

[27] Tianyi Wang, "Bearing life prediction based on vibration signals: A case study and lessons learned", IEEE Conference on Prognostics and Health Management, Beijing, 2012. DOI: 10.1109/ICPHM.2012.6299547.

[28] Yuanhong Chang, Jinglong Chen, Haixin Lv, and Shen Liu, "Heterogeneous bi-directional recurrent neural network combining fusion health indicator for predictive analytics of rotating machinery", ISA Transactions, 2021. DOI: 10.1016/j.isatra.2021.04.024.

[29] Khanh T. P. Nguyen and Kamal Medjaher, "An automated health indicator construction methodology for prognostics based on multi-criteria optimization", ISA Transactions, Vol. 113, pp. 81 - 96, 2021. DOI: 10.1016/j. isatra.2020.03.017.

[30] Yaguo Lei, Naipeng Li, Liang Guo, Ningbo Li, Tao Yan, and Jing Lin, "Machinery health prognostics: A systematic review from data acquisition to RUL prediction", Mechanical Systems and Signal Processing, Vol. 104, pp. 799 - 834, 2018. DOI: 10.1016/j.ymssp.2017.11.016.

[31] Jay Lee, Hai Qiu, Jing Lin, and Gang Yu, "Robust performance degradation assessment methods for enhanced rolling element bearing prognostics", Advanced Engineering Informatics, Vol. 17, No. 3 - 4, pp. 127 - 140, 2003. DOI: 10.1016/j.aei.2004.08.001.

[32] Zheng Zhou, Sheng Hong, Enrico Zio, and Kan Hong, "Condition assessment for the performance degradation of bearing based on a combinatorial feature extraction method", Digital Signal Processing, Vol. 27, pp. 159 - 166, 2014. DOI: 10.1016/j.dsp.2013.12.010.

[33] Yaguo Lei, Naipeng Li, Szymon Gontarz, Jing Lin, Stanislaw Radkowski, and Jacek Dybala, "A model-based method for remaining useful life prediction of machinery", IEEE Transactions on Reliability, Vol. 65, No. 3, pp. 1314 1326, 2016. DOI: 10.1109/TR.2016.2570568.

[34] Linxia Liao, Radu Pavel, and Wenjing Jin, "Enhanced restricted Boltzmann machine with prognosability regularization for prognostics and health assessment", IEEE Transactions on Industrial, Vol. 63, No. 11, pp. 7076 - 7083, 2016. DOI: 10.1109/TIE.2016.2586442.

[35] Runqing Huang, Lifeng Xi, Xinglin Li, C. Richard Liu, Hai Qiu, and Jay Lee, "Residual life predictions for ball bearings based on self-organizing map and back propagation neural network methods", Mechanical Systems and Signal Processing, Vol. 21, No. 1, pp. 193 - 207, 2007. DOI: 10.1016/j.ymssp.2005.11.008.

[36] Yu Wang, Yizhen Peng, Zi, Yanyang Zi, Xiaohang Jin, and Kwok-Leung Tsui, "A two-stage data-driven-based prognostic approach for bearing degradation problem", IEEE Transactions on Industrial Informatics, Vol. 12, No. 3, pp. 924 - 932, 2016. DOI: 10.1109/TII.2016.2535368.

[37] Xiaohang Jin, Yi Sun, Zijun Que, Yu Wang, and Tommy Wai Shing Chow, "Anomaly detection 
and fault prognosis for bearings", IEEE Transactions on Instrumentation and Measurement, Vol. 65, pp. 2046 - 2054, 2016. DOI: 10.1109/TIM.2016.2570398.

[38] Kumar H S, P. Srinivasa Pai, N. S. Sriram, and Vijay Gs, "Rolling element bearing fault diagnostics: Development of health index", Proceedings of the Institution of Mechanical Engineers, Part C: Journal of Mechanical Engineering Science 1989 - 1996, Vol. 231, No. 21, pp. 3923 3939, 2017. DOI: $10.1177 / 0954406216656214$.

[39] Hasan Ocak, Kenneth A. Loparo, Fred M. Discenzo, "Online tracking of bearing wear using wavelet packet decomposition and probabilistic modeling: A method for bearing prognostics", Journal of Sound and Vibration, Vol. 302, No. 4 - 5, pp. 951 - 961, 2007. DOI: 10.1016/j.jsv.2007.01.001.

[40] Huseyin M. Ertunc, Kenneth A. Loparo, Hasan Ocak, "Tool wear condition monitoring in drilling operations using hidden Markov models (HMMs)", International Journal of Machine Tools and Manufacture, Vol. 41, No. 9, pp. 1363 - 1384, 2001. DOI: 10.1016/S08906955(00)00112-7.

[41] Hasan Ocak and Kenneth A. Loparo, "HMMbased fault detection and diagnosis scheme for rolling element bearings", Journal of Vibration and Acoustics, Vol. 127, No. 4, pp. 299 - 306, 2005. DOI: 10.1115/1.1924636.

[42] Zhongjjie Shen, Zhengjja He, Xuefeng Chen, Chuang Sun, and Zhiwen Liu, "A monotonic degradation assessment index of rolling bearings using fuzzy support vector data description and running time", Sensors, Vol. 12, No. 8, pp. 10109 - 10135, 2012. DOI: 10.3390/s120810109.

[43] Yan-Cheng Liu, Siyuan Liu, and Ning Wang, "Fully-tuned fuzzy neural network based robust adaptive tracking control of unmanned underwater vehicle with thruster dynamics", Neurocomputing, Vol. 196, pp. 1 - 13, 2016. DOI: 10.1016/j.neucom.2016.02.042.

[44] Guo Liang, Li Naipeng, Jia Feng, Lei Yaguo, and Lin Jing, "A recurrent neural network based health indicator for remaining useful life prediction of bearings", Neurocomputing, Vol. 240, pp. 98 - 109, 2017. DOI: 10.106/j. neucom.2017.02.045.

[45] Emmanuel Ramasso, Michèle Rombaut, and Noureddine Zerhouni, "Joint prediction of continuous and discrete states in time-series based on belief functions", IEEE Transactions on Cybernetics, Vol. 43, No. 1, pp. 37 - 50, 2013.
[46] Kamran Javed, Rafael Gouriveau, and Noureddine Zerhouni, "A new multivariate approach for prognostics based on extreme learning machine and fuzzy clustering", IEEE Transactions on Cybernetics, Vol. 45, No. 12, pp. 2626 - 2639, 2015. DOI: 10.1109/TCYB.2014.2378056.C

[47] Zhilian Liu, Ming J. Zuo, and Yong Qin, "Remaining useful life prediction of rolling element bearings based on health state assessment", Proceedings of the Institution of Mechanical Engineers, Part C: Journal of Mechanical Engineering Science, Vol. 230, No. 2, pp. 314 330, 2016. DOI: $10.1177 / 0954406215590167$.

[48] Patricia Scanlon, Darren F. Kavanagh, and Francis Boland, "Residual life prediction of rotating machines using acoustic noise signals", IEEE Transactions on Instrumentation and Measurement, Vol. 62, pp. 95 - 108, 2012. DOI: 10.1109/TIM.2012.2212508.

[49] Andrea Giantomassi, Francesco Ferracuti, Alessandro Benini, Gianluca Ippoliti, Sauro Longhi, and Antonio Petrucci, "Hidden Markov model for health estimation and prognosis of turbofan engines", ASME 2011 International Design Engineering Technical Conferences and Computers and Information in Engineering Conference, 28 31 August, 2011. DOI: 10.1115/DETC2011-48174.

[50] E. Ramasso and T. Denoeux, "Making use of partial knowledge about hidden states in HMMs: An approach based on belief functions", IEEE Transactions on Fuzzy Systems, Vol. 22, pp. 395 - 405, 2014.

[51] F. Sloukia, R. Bouarfa, H. Medromi, and M. Wahbi, "Bearings prognostic using mixture of Gaussians hidden Markov model and support vector machine", International Journal of Network Security \& Its Applications, Vol. 5, pp. 85 - 97, 2013.

[52] P. Tamilselvan, Yibin Wang, and P. Wang, "Deep belief network based state classification for structural health diagnosis", 2012 IEEE Aerospace Conference, Montana, 3 - 10 March, 2012.

[53] Liang Guo, Hongli Gao, Haifeng Huang, Xiang $\mathrm{He}$, and ShiChao Li, "Multifeatures fusion and non-linear dimension reduction for intelligent bearing condition monitoring", Shock and Vibration, Vol. 2016, pp. 1 - 10, 2016. DOI: 10.1155/2016/4632562.

[54] Jaouher Ben Ali, Brigitte Chebel-Morello, Lotfi Saidi, Simon Malinowski, and Farhat Fnaiech, "Accurate bearing remaining useful life prediction based on Weibull distribution and artificial neural network", Mechanical 
Systems and Signal Processing, Vol. 56 - 57, pp. 150 - 172, 2015.

[55] A. Soualhi, K. Medjaher, and N. Zerhouni, "Bearing health monitoring based on Hilber-Huang transform, support vector machine, and regression", IEEE Transactions on Instrumentation and Measurement, Vol. 64, pp. 52 - 62, 2015.

[56] Hack-Eun Kim, Andy C.C. Tan, Joseph Mathew, and Byeong-Keun Choi, "Bearing fault prognosis based on health state probability estimation", Expert Systems with Application, Vol. 39, pp. 5200 - 5213, 2012.

[57] Xiao-Sheng Si, Wenbin Wang, Chang-Hua Hu, Dong-Hua Zhou, "Remaining useful life estimation - A review on the statistical data driven approaches", European Journal of Operational Research, Vol. 213, pp. 1 - 14, 2011.

[58] Y. Li, S. Billington, C. Zhang, T. Kurfess, S. Danyluk, and S. Liang, "Adaptive prognostics for rolling element bearing condition", Mechanical Systems and Signal Processing, Vol. 13, No. 1, pp. 103 - 113, 1999.

[59] Yaguo Lei, Naipe Li, and Jing Lin, "A new method based on stochastic process models for machine remaining useful life prediction", IEEE Transactions on Instrumentation and Measurement, Vol. 65, pp. 2671 - 2684, 2016.

[60] C. Sbarufatti, M. Corbetta, A. Manes, and M. Giglio, "Sequential Monte-Carlo sampling based on a committee of artificial neural networks for posterior state estimation and residual lifetime prediction", International Journal of Fatigue, Vol. 83, pp. 10 - 23, 2016.

[61] Yongping Pan, Meng Joo Er, Xiang Li, Haoyong Yu, and Rafael Gouriveau, "Machine health condition prediction via online dynamic fuzzy neural networks", Engineering Applications of Artificial Intelligence, Vol. 35, pp. 105 - 113, 2014.

[62] Lei Xiao, Xiaohui Chen, Xinghui Zhang, and Min Liu, "A novel approach for bearing remaining useful life estimation under neither failure nor suspension histories condition", Journal of Intelligent Manufacturing, Vol. 28, pp. 1893 - 1914, 2017. DOI: 10.1007/s10845-015-1077-x.

[63] Lu Wang, Li Wang, Xue-zhi Wang, "Reliability estimation and remaining useful lifetime prediction for bearing based on proportional hazard model", Journal of Central South University, Vol. 22, pp. 4625 - 4633, 2015.

[64] Ryad Zemouri and Rafael Gouriveau, "Towards accurate and reproducible predictions for prognostic:
An approach combining a RRBF network and an autoRegressive model", IFAC Proceedings Volumes, Vol. 43, No. 3, pp. 140 - 145, 2010.

[65] Arnaz Malhi, Ruqiang Yan, and Robert X. Gao, "Prognosis of defect propagation based on recurrent neural networks", IEEE Transactions on Instrumentation and Measurement, Vol. 60, pp. 703 - 711, 2011. DOI: 10.1109/ TIM.2010.2078296.

[66] Yu Peng, Hong Wang, Jianmin Wang, Datong Liu, and Xiyuan Peng, "A modified echo state network based remaining useful life estimation approach", 2012 IEEE Conference on Prognostics and Health Management, 2012.

[67] Datong Liu, Wei Xie, Haitao Liao, and Yu Peng, "An integrated probabilistic approach to Lithium-lon battery remaining useful life estimation", IEEE Transactions on Instrumentation and Measurement, Vol. 64, pp. 660 670, 2015.

[68] IBM Cloud Education, "Recurrent neural networks", 2020. [Online]. Available: https://www.ibm. com/cloud/learn/recurrent-neural-networks.

[69] Samarjit Kar, Sujit Das, and Pijush Ghosh, "Applications of neuro fuzzy systems: A brief review and future outline", Applied Soft Computing, Vol. 15, pp. 243 259, 2014.

[70] Jyh-Shing Roger Jang, Chuen-Tsai Sun, and Eiji Mizutani, Neuro-fuzzy and soft computing: A computational approach to learning and machine intelligence. PrenticeHall Inc., 1996.

[71] Wilson Q. Wang, M. Farid Golnaraghi, and Fathy Ismail, "Prognosis of machine health condition using neuro-fuzzy systems", Mechanical Systems and Signal Processing, Vol. 18, No. 4, pp. 813 - 831, 2004. DOI: 10.1016/ s0888-3270(03)00079-7.

[72] Wilson Wang, "An adaptive predictor for dynamic system forecasting", Mechanical Systems and Signal Processing, Vol. 21, No. 2, pp. 809 - 823, 2007. DOI:10.1016/j.ymssp.2005.12.008.

[73] Fagang Zhao, Jin Chen, Lei Guo, and Xinglin $\mathrm{Li}$, "Neuro-fuzzy based condition prediction of bearing health", Journal of Vibration and Control, Vol. 15, No. 7, pp. 1079 - 1091, 2009. DOI:10.1177/1077546309102665.

[74] Van Tung Tran, Bo-Suk Yang, and Andy Chit Chiow Tan, "Multi-step ahead direct prediction for the machine condition prognosis using regression trees and 
neuro-fuzzy systems", Expert Systems with Applications, Vol. 36, No. 5, pp. 9378 - 9387, 2009. DOI: 10.1016/j. eswa.2009.01.007

[75] Chaochao Chen, Bin Zhang, George Vachtsevanos, and Marcos E.Orchard, "Machine condition prediction based on adaptive neuro-fuzzy and high-order particle filtering", IEEE Transactions on Industrial Electronics, Vol. 58, No. 9, pp. 4353 - 4364, 2011.

[76] Daniel Zurita Millan, Jesus A.Carino, Miguel Delgado-Prieto, and Juan Antonio Redondo, "Distributed neuro-fuzzy feature forecasting approach for condition monitoring", 2014 IEEE Emerging Technology and Factory Automation (ETFA), 2015.

[77] Sajid Hussain and Hossam A.Gabbar, "Vibration analysis and time series prediction for wind turbine gearbox prognostics", International Journal of Prognostics and Health Management, Vol. 4, No. 3, 2013. DOI: 10.36001/ ijphm.2013.v4i3.2144.

[78] Vladimir N.Vapnik, "An overview of statistical learning theory", IEEE transactions on neural networks, Vol. 10, No. 5, pp. 988 - 999, 1999.

[79] Shaojiang Dong and Tianhong Luo, "Bearing degradation process prediction based on the PCA and optimized LS-SVM model", Measurement, Vol. 46, No. 9, pp. 3143 - 3152, 2013. DOI: 10.1016/j. measurement.2013.06.038.

[80] J. Carino, D. Zurita, Miguel Delgado Prieto, J. A. Redondo, and R. Romero-Troncoso, "Remaining useful life estimation of ball bearings by means of monotonic score calibration", IEEE International Conference on Industrial Technology (ICIT), 17 - 19 March 2015. DOI: 10.1109/ ICIT.2015.7125351.

[81] James K. Kimotho, Christoph SondermannWoelke, Tobias Meyer, and Walter Sextro, "Machinery prognostic method based on multi-class support vector machines and hybrid differential evolution particle swarm optimization", Chemical Engineering Transactions, Vol. 33, pp. $619-624,2013$.

[82] Achmad Widodo and Bo-Suk Yang, "Machine health prognostics using survival probability and support vector machine", Expert Systems with Applications, Vol. 38, No. 7, pp. 8430 - 8437, 2011. DOI: 10.1016/j. eswa.2011.01.038.
[83] Van Tung Tran and Bo-Suk Yang, "An intelligent condition-based maintenance platform for rotating machinery", Expert Systems with Applications, Vol. 39, No. 3, pp. 2977 - 2988, 2012. DOI:10.1016/j.eswa.2011.08.159.

[84] T. Benkedjouh, K. Medjaher, N. Zerhouni, and S. Rechak, "Health assessment and life prediction of cutting tools based on support vector regression", Journal of Intelligent Manufacturing, Vol. 26, pp. 213 - 223, 2015. DOI: 10.1007/s10845-013-0774-6.

[85] Jie Liu and Enrico Zio, "An adaptive online learning approach for support vector regression: onlineSVR-FID", Mechanical Systems and Signal Processing, Vol. 76, pp. 796 - 809, 2016. DOI: 10.1016/j.ymssp.2016.02.056.

[86] Emanuele Fumeo, Luca Oneto, Davide Anguita, "Condition based maintenance in railway transportation systems based on big data streaming analysis", Procedia Computer Science, Vol. 53, pp. 437 - 446, 2015. DOI: 10.1016/j.procs.2015.07.321.

[87] W. J. Padgett and Meredith A. Tomlinson, "Inference from accelerated degradation and failure data based on Gaussian process models", Lifetime Data Analysis, Vol. 10, pp. 191 - 206, 2004.

[88] Carl Edward Rasmussen, "Gaussian processes in machine learning", Advanced Lectures on Machine Learning, pp. 63 - 71, 2003. DOI: 10.1007/978-3-540-28650-9_4.

[89] Kai Goebel, Bhaskar Saha, and Abhinav Saxena, "A comparison of three data-driven techniques for prognostics", 2008.

[90] S.A. Aye, P.S. Heyns, "An integrated Gaussian process regression for prediction of remaining useful life of slow speed bearings based on acoustic emission", Mechanical Systems and Signal Processing, Vol. 84, pp. 485 498, 2017. DOI: 10.1016/j.ymssp.2016.07.039.

[91] Man Shan Kan, Andy C.C. Tan, and Joseph Mathew, "A review on prognostic techniques for nonstationary and non-linear rotating systems", Mechanical Systems and Signal Processing, Vol. 62 - 63, pp. 1 - 20, 2015. DOI: $10.1016 /$ j.ymssp.2015.02.016 